\title{
Neural regulation of discontinuous gas exchange in Periplaneta americana
}

\author{
James D. Woodman ${ }^{\mathrm{a}, \mathrm{b}, *}$, Paul D. Cooper ${ }^{\mathrm{a}}$, Victoria S. Haritos ${ }^{\mathrm{b}}$ \\ ${ }^{a}$ School of Botany and Zoology, Australian National University, Canberra ACT 0200, Australia \\ ${ }^{\mathrm{b}}$ CSIRO Entomology, GPO Box 1700, Canberra ACT 2601, Australia
}

Received 28 September 2007; received in revised form 22 November 2007; accepted 26 November 2007

\begin{abstract}
Patterns of gas exchange among terrestrial arthropods are highly variable from continuous to discontinuous with discretely partitioned phases. The underlying initiation and co-ordination of these patterns is relatively poorly understood. Here we present a novel method for the simultaneous measurement of central nervous system (CNS) activity of the metathoracic ganglion and $V \mathrm{CO}_{2}$ in medium to large sized live terrestrial arthropods. Using Periplaneta americana at four oxygen levels $\left(40 \%, 21 \%, 10 \%\right.$ and $2 \%$ at $25^{\circ} \mathrm{C} ; n=6$ per treatment), we present minimally invasive visualization of nervous output relative to typical resting discontinuous gas exchange (DGE) data for the first time. DGE was maintained when cockroaches were exposed to hyperoxia or moderate hypoxia, but was lost in severe hypoxia. CNS activity was manifested in three signal types: large CNS output coinciding with peak $\mathrm{CO}_{2}$ production during a burst, moderate CNS output coinciding with $\mathrm{CO}_{2}$ sawtoothing and fluttering, and minimal CNS activity during the closed phase of DGE in normoxia. Large and moderate CNS outputs were associated with observed abdominal pumping and congruent $\mathrm{CO}_{2}$ peaks. At $10 \%$ oxygen, $V C \mathrm{O}_{2}$ was significantly elevated during the inter-burst period in association with almost constant moderate CNS output between the periodic large CNS output. At $2 \%$ oxygen, DGE and large CNS output are lost to continuous $\mathrm{CO}_{2}$ release and largely continuous moderate CNS output. As previously reported for this species, a central pattern generator for ventilation in the metathoracic ganglion is supported and we infer the presence of localized oxygen chemoreceptors based on clear CNS response to a change in oxygen tension.
\end{abstract}

(C) 2007 Elsevier Ltd. All rights reserved.

Keywords: CNS; Discontinuous gas exchange; Oxygen tension; Respirometry; Metathoracic ganglion

\section{Introduction}

Efficient gas exchange in most terrestrial arthropods is reliant on the tracheal system and adequate ventilation. Ventilation may be achieved by passive diffusion or may involve active muscular movements and selective opening of the spiracles (Wasserthal, 1996; Lighton, 1996; Slama, 1999; Chown et al., 2006). Patterns of gas exchange vary considerably within and among species relative to factors, including body size and atmospheric oxygen concentration. Whilst various gas exchange patterns have been described, including continuous, cyclic, and discontinuous gas exchange (DGE, e.g. Marais and Chown, 2003), the

\footnotetext{
${ }^{*}$ Corresponding author at: School of Botany and Zoology, Australian National University, Canberra ACT 0200, Australia.

Tel.: + 61262464031.

E-mail address: james.woodman@csiro.au (J.D. Woodman).
}

underlying generation and co-ordination of these patterns remain relatively poorly understood (Chown et al., 2006).

Among terrestrial insects, a major distinction can be made between ventilation during periods of locomotion or flight, where high-frequency continuous gas exchange is typically observed, and during periods of rest, which may be typified by reduced frequency DGE (Reinhold, 1999). Discontinuous or cyclic ventilation patterns have been recorded for a number of insects during quiescence, whereby $\mathrm{CO}_{2}$ release bursts are separated by inter-burst periods that may incorporate a closed spiracle phase and a flutter phase, where the spiracles rapidly open and close to regulate tracheal oxygen levels (Lighton, 1996; Chown et al., 2006; Quinlan and Gibbs, 2006). The nervous activity, which initiates and maintains discontinuous ventilation, is of considerable interest as it is likely to provide a clearer understanding of the function of DGE. 
The motor activity that drives active insect ventilation is dependent on patterned neural control generated by a respiratory pacemaker or central pattern generator $(\mathrm{CPG}$, review: Marder and Bucher, 2001). In locusts, ventilatory rhythm is generated primarily in the metathoracic ganglion of the central nervous system (CNS; Miller, 1960; Bustami and Hustert, 2000). The metathoracic ganglion has also been suggested as a primary $\mathrm{CPG}$ in the American cockroach, Periplaneta americana, despite evidence for shifting or multiple CPGs (Farley et al., 1967). The coordination of ventilatory movements among body segments is achieved by a CPG in one segment imposing a rhythm on other segments via linking interneurones (Lewis et al., 1973; Burrows, 1975, 1982). These interneurones, some of which have been identified in locusts, communicate the rhythm to the abdominal ganglia and subsequently the motor neurons, which innervate the relevant muscles for abdominal pumping and spiracular control.

The ventilatory output from a CPG may vary widely within a species and even within a single individual, depending on many factors (Bustami et al., 2002). For example, oxygen tension has been shown to affect gas exchange for a range of species and more specifically, directly impact on CNS ventilatory output in locusts and dragonflies (Miller, 1960, 1966; Mill and Hughes, 1966). The presence of chemoreceptors sensitive to oxygen concentration, most probably located within the metathoracic ganglion, has been confirmed for the horse lubber grasshopper, Taeniopoda eques (Bustami et al., 2002). It was shown that CNS output bursts per minute from the isolated ganglion submerged in saline varied with changes in oxygen tension. When exposed to anoxia, a biphasic response was recorded whereby a dramatic, but transient increase in ventilatory nervous output was followed by total inactivity.

To our knowledge, no previous study has examined live, intact specimens shown to be performing a typical DGE pattern at the time of nervous recording. Previous research has mostly relied on various dissection techniques and the use of isolated sections of the CNS (Myers and Retzlaff, 1963; Farley et al., 1967; Ramirez and Pearson, 1989; Burrows, 1982; Bustami and Hustert, 2000). Whilst isolated sections submerged in saline may provide reliable rhythmic patterns theoretically void of any sensory or behavioral disturbance for up to several hours, it is nevertheless an invasive and damaging technique (Bustami and Hustert, 2000; Zafeiridou and Theophilidis, 2006). The potential for increased noise in the recordings and abnormal ventilation patterns as a result of the dissecting process has long been recognized as an experimental limitation that is difficult to fully reconcile.

Given this background, we recognized potential in being able to describe how DGE is regulated by CNS output in live animals. Specifically, we wanted to describe the ventilatory nervous output from the CNS associated with individual DGE phases and visualize responses to different oxygen tensions. Here we report a novel technique to address these aims which permits simultaneous measurement of ventilatory nervous activity from the intact CNS and $V \mathrm{CO}_{2}$ from live specimens. Using $P$. americana, we use this method to gain functional insight into the regulation of DGE in insects and the CNS output associated with ventilation, including $\mathrm{CO}_{2}$ bursts from the spiracles. We use normoxic as well as hypoxic ( $2 \%$ and $10 \%$ oxygen) and hyperoxic ( $40 \%$ oxygen) treatments to report how low and high oxygen levels affect the normal resting gas exchange pattern. Respirometry-only experiments without electrode placement for CNS measurement are used to validate data from the simultaneous CNS measurements trials.

\section{Materials and methods}

\subsection{Study species}

The American cockroach, $P$. americana, performs DGE (Kestler, 1985) and has been the subject of previous neurophysiological research identifying the metathoracic ganglion as a respiratory pacemaker (Farley et al., 1967). Adult males were obtained from established breeding colonies maintained at the Australian National University.

\subsection{Recording ventilatory activity from the intact CNS}

Animals were weighed using an analytical balance (sensitivity $0.1 \mathrm{mg}$; HR-200, A\&D Co., Tokyo, Japan) and cooled to $4{ }^{\circ} \mathrm{C}$ to render them immobile. As required, each specimen was transferred onto ice (positioned ventral side up, posterior facing away) for the removal of the second leg from the left side to provide lateral access to the CNS. The animal was then transferred into a custom-built vertical cylindrical screw-top Perspex chamber (internal dimensions: radius $45 \mathrm{~mm}$, height $55 \mathrm{~mm}$, volume $350 \mathrm{~cm}^{3}$ ), where it was centrally positioned on its back and held in place with a strip of flexible adhesive over the three legs on the right side (Fig. 1). Paired platinum hook electrodes $0.5 \mathrm{~mm}$ in diameter were inserted from the side of the chamber on a rigid stalk. The insertion through a rubber seal permitted subsequent fine positioning by micromanipulators. The electrodes were positioned to the left of the paired nerve cord and curved forceps were used to pierce through the unsclerotized, flexible exoskeleton on each side. From the right, the paired central nerve cords were gently lifted onto the electrodes which were slightly bent upwards at the tip to prevent slipping during a trial. After positioning anteriorly, so as to abut the metathoracic ganglion, the electrodes were raised very slightly to ensure nerve contact and left in place for the duration of the recording. The lid of the chamber was fastened immediately using high vacuum grease to seal the chamber.

The electrodes were connected to an amplifier (Powerlab 4/25T; ADInstruments, Bella Vista, Australia), which was connected to a personal computer. Data acquisition software (Chart v.5.2.2; ADInstruments, Bella Vista, Australia) was used to record electrical output at a 


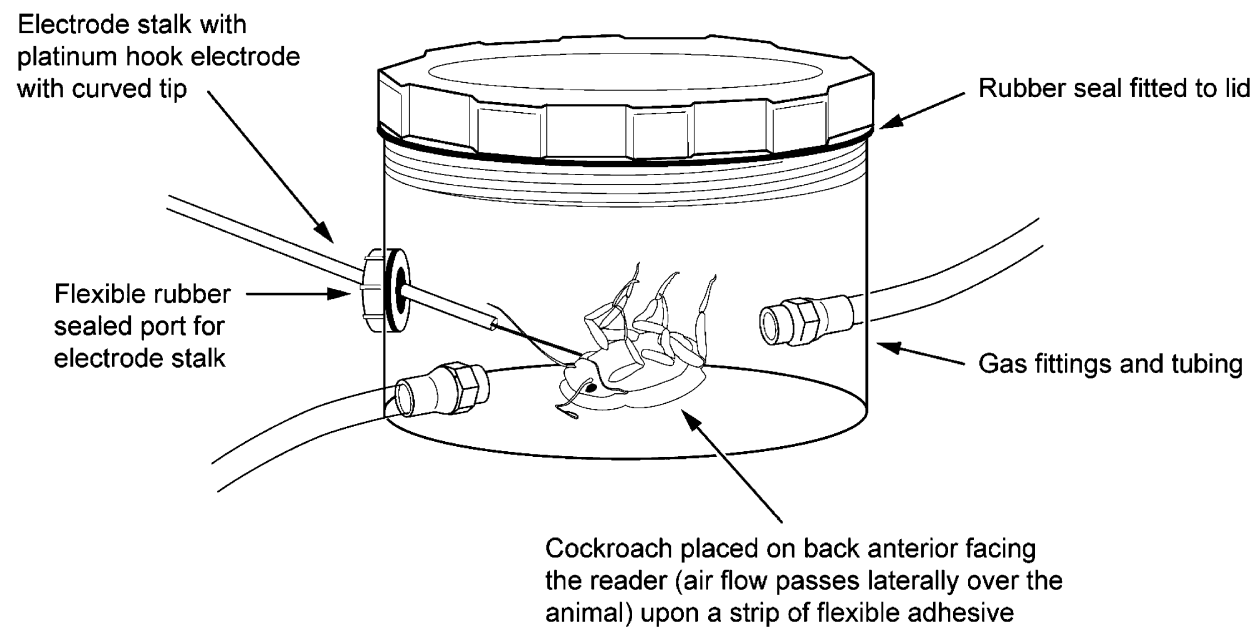

Fig. 1. An annotated diagrammatic representation of the vertical cylindrical respirometry chamber and electrode used for simultaneous measurement of ventilatory CNS activity and gas exchange.

frequency of 10 measurement $\mathrm{s}^{-1}$. Data were subsequently transferred into Microsoft Excel for analysis.

\subsection{Recording gas exchange}

The recording of $\mathrm{CO}_{2}$ output for each individual was achieved by the incorporation of the sealed chamber into a flow-through respirometry system using a calibrated Licor 7000 differential infrared gas analyzer (Li-Cor, Lincoln, USA). The input and output tubing (Bev-a-line IV) was connected to opposing gas fittings on the side of the chamber (Fig. 1) and gas was drawn through the system by an Edwards E2M-1.5 high-vacuum pump (BOC-Edwards, BOC group Inc., Sussex, England). Experiments proceeded with either $40 \%, 21 \%, 10 \%$ or $2 \%$ oxygen in nitrogen passing through the chamber at a rate of $250 \mathrm{~mL} \mathrm{~min}^{-1}$ (time constant of the chamber $=84 \mathrm{~s}$, see Gray and Bradley, 2006). The experimental gas mixtures were achieved by mixing high-purity oxygen and nitrogen, which passed through an inline moisture and $\mathrm{CO}_{2}$ removal column (Glass moisture trap, Model 7214; Alltech, Australia). The ratio of each gas used was controlled by a Brooks 5878 mass-flow controller with three Brooks 5850TR mass-flow meters, whose calibrations were checked before use with a calibrated digital flow meter (one each for vacuum, $\mathrm{O}_{2}$, and $\mathrm{N}_{2}$ ) at $0-500 \mathrm{~mL} \mathrm{~min}^{-1}$ (Brooks Instruments, Hatfield, Pennsylvania, USA). The use of mass-flow controllers permitted the gas composition to be instantaneously varied as required without disruption to the gas line.

Raw data from the gas analyzer were logged on to a second personal computer using Licor LI7000 data acquisition software (Version 1.0.1). The lag time between the nervous and $\mathrm{CO}_{2}$ recordings was negligible due to the simultaneous initiation of both data acquisition programs. The data from each trial were imported into Microsoft Excel for analysis with the CNS data.

\subsection{Treatments}

Cockroaches were observed periodically during all experiments for visible ventilatory and gross bodily movements and the timing of each was recorded.

Standard patterns of gas exchange and ventilatory nervous output were recorded for $P$. americana at $21 \%$ oxygen in nitrogen over a $5 \mathrm{~h}$ period. For measuring the effects of different oxygen concentrations, oxygen tensions of $2 \%, 10 \%$ and $40 \%$ were used. All trials were conducted under dim light at $25 \pm 1{ }^{\circ} \mathrm{C}(n=6$ individual animals per treatment). For the three experimental oxygen tensions, each specimen was exposed to $2 \mathrm{~h}$ of $21 \%$ oxygen in nitrogen, followed by $2 \mathrm{~h}$ of the experimental treatment and then a further $1 \mathrm{~h}$ of $21 \%$.

To validate the data during CNS measurement we also conducted a series of respirometry-only experiments at each of the previously mentioned oxygen tensions using the same chamber as that used in the CNS measurement experiments. Experiments proceeded under dim light at $25 \pm 1{ }^{\circ} \mathrm{C} \quad(n=6$ individual animals per treatment) as described for the CNS measurement trials (including the cockroaches being overturned and held in place with the second leg on the left side removed); however, there was no dissection or electrode placement. Exposure to each oxygen tension was maintained for at least $2 \mathrm{~h}$.

\subsection{Data analysis and definitions}

DGE cycles were selected from the $\mathrm{CO}_{2}$ output data from each experiment ( $n=2-8$ cycles per animal). The first $30 \mathrm{~min}$ of recording in each trial was discarded due to elevated $\mathrm{CO}_{2}$ output while the animal settled. Periods of gross movement (as opposed to abdominal ventilatory movement) were identified from direct observation as well as perturbation in $V \mathrm{CO}_{2}$ and excluded from analysis. In the 
absence of DGE at $2 \%$ oxygen, $\mathrm{CO}_{2}$ output rates were calculated using a $1 \mathrm{~h}$ period for each individual whilst at rest. To be considered as a DGE cycle for inclusion in analysis, a cycle had to exhibit an inter-burst period, whereby low $\mathrm{CO}_{2}$ output was maintained, followed by a clearly defined $\mathrm{CO}_{2}$ burst phase, as typified by DGE cycles

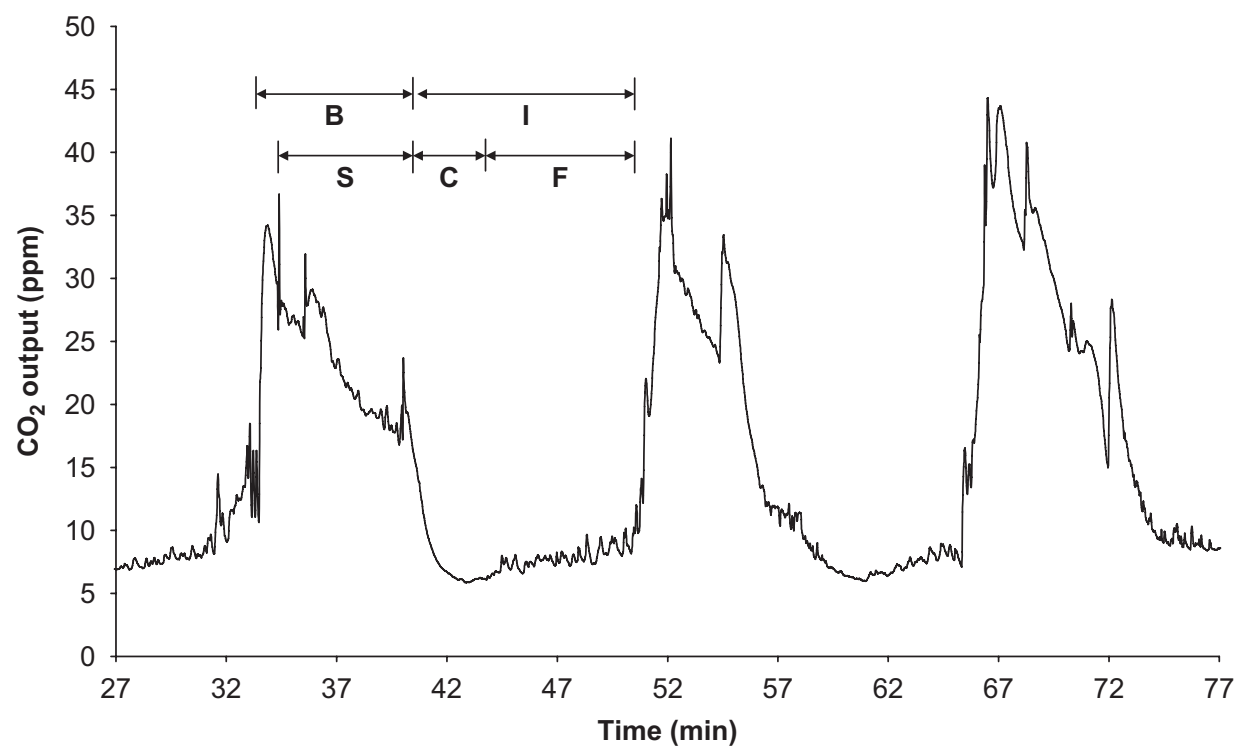

Fig. 2. Representative respirometry-only (without CNS recording) results for $P$. americana at normoxia at $25^{\circ} \mathrm{C}$. Animals were overturned and restrained as per the preparation used for simultaneous CNS measurements, but without electrode placement. The gas exchange pattern was separated into a burst phase (B) incorporating sawtoothing (S) and inter-burst phase (I) incorporating the closed (C) and flutter (F) periods.

a

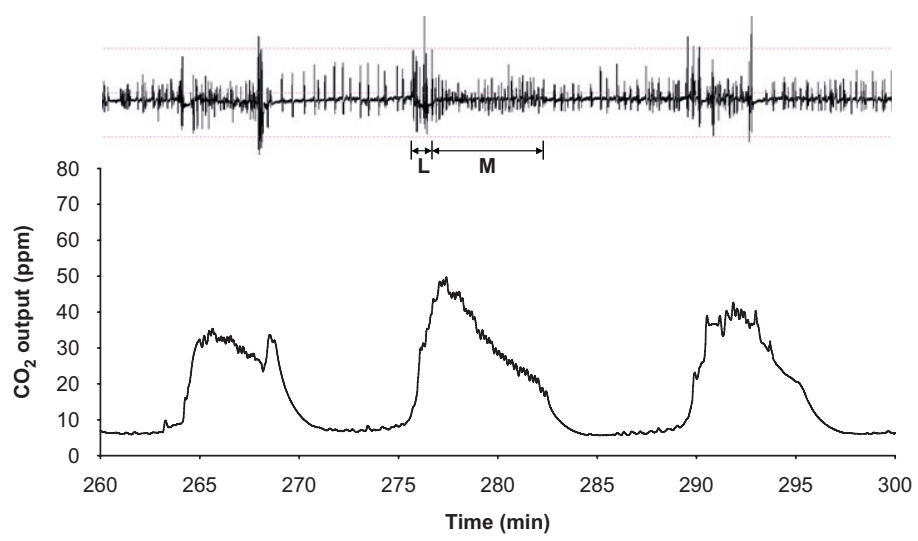

C

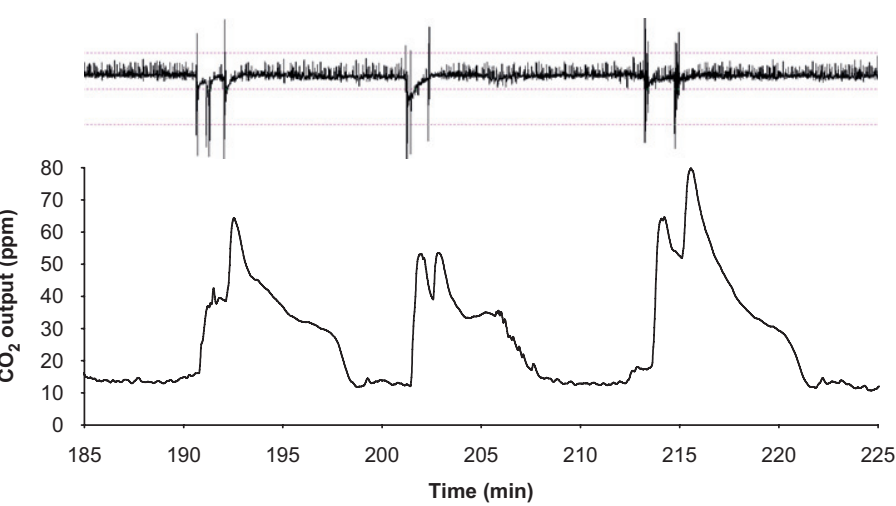

b

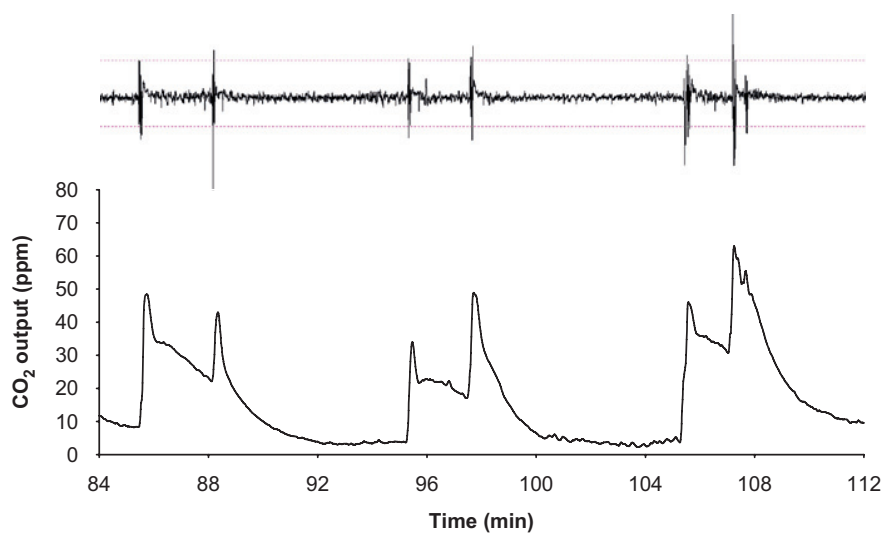

d
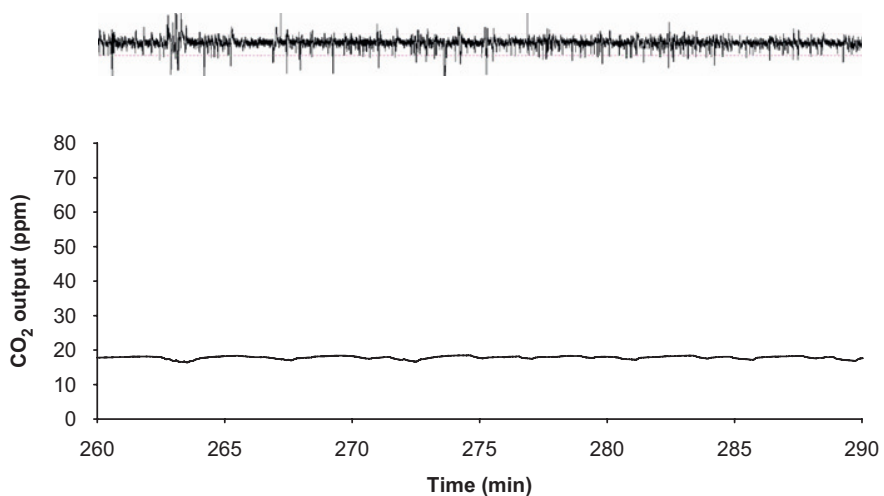

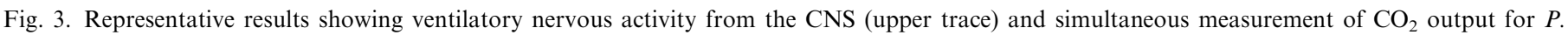
americana over $28-40 \mathrm{~min}$ (to show 3 DGE cycles) at (a) normoxia- $21 \%$ oxygen, (b) hyperoxia $-40 \%$ oxygen, (c) moderate hypoxia- $10 \%$ oxygen, and (d) severe hypoxia- $2 \%$ oxygen. Examples of large (L) and moderate (M) CNS output are shown in trace (a). 
previously presented for P. americana in Kestler (1985) and demonstrated here in Fig. 2.

CNS activity corresponding to $V \mathrm{CO}_{2}$ was analyzed, whereby only clear CNS output that matched the gas exchange data was used, as demonstrated in Fig. 3. The largest CNS activity was defined by the highest amplitude CNS outputs associated with $\mathrm{CO}_{2}$ bursts during DGE. Moderate CNS activity was defined as a series of outputs of similar amplitude, approximately $20-30 \%$ of the amplitude of large CNS output (Fig. 3(a)). Minimal CNS output relative to the baseline was observed only transiently at normoxia during the inter-burst period, when it was assumed that some of the spiracles might be closed and muscular ventilation relaxed (Fig. 3(a)).

\section{Results}

Measurement of ventilatory CNS activity from the metathoracic ganglion in $P$. americana, whilst simultaneously recording $V \mathrm{CO}_{2}$ for up to $5 \mathrm{~h}$, was consistent and repeatable. Lively specimen condition in excess of 3 days after a trial confirms that there was minimal damage to the cockroaches due to the procedure and indicates that an individual could be repeatedly measured over an extended period if required. We limited the recording of nervous output and $V \mathrm{CO}_{2}$ to $5 \mathrm{~h}$ periods due to drying of the contact between the electrode and the ganglion and gradual loss of CNS signal fidelity after that time. By using a flow rate of $250 \mathrm{~mL} \mathrm{~min}^{-1}$ in a relatively large chamber we minimized dehydration of the exposed ganglion, but maintained sufficient $\mathrm{CO}_{2}$ measurement fidelity to clearly distinguish different gas exchange patterns as well as the phases of the DGE cycle. A stable zero baseline was achieved for both $\mathrm{CO}_{2}$ and water output before commencing each experiment; however, slightly elevated $\mathrm{CO}_{2}$ output was observed during the inter-burst period of DGE compared with that previously measured for P. americana (Fig. 3; Kestler, 1985). This is most likely associated with either flow rate or the experimental preparation, but does not compromise examination of the correlation between CNS activity and gas exchange. Importantly, after each animal was removed from the chamber, $\mathrm{CO}_{2}$ and water output measurements consistently returned to a stable zero baseline.

\subsection{Ventilation in resting animals}

The typical gas exchange pattern of adult $P$. americana when immobile is DGE, featuring three phases (Fig. 2; Kestler, 1985). There is a clearly distinguished $\mathrm{CO}_{2}$ burst phase, which represents a co-ordinated opening of the spiracles and abdominal pumping to convey a bulk discharge of $\mathrm{CO}_{2}$ from the body. A closed phase has the lowest $\mathrm{CO}_{2}$ release of the cycle and is considered a period where the spiracles are mostly closed. The flutter phase follows the closed phase and represents small releases of $\mathrm{CO}_{2}$, consistent with rapidly opening and closing the spiracles to most probably draw air inward to increase oxygen levels (Kestler, 1985; Lighton, 1996). Respirometry-only experiments using an overturned animal held in place, but without dissection or electrode placement, gave a standard three-phase DGE response (Fig. 2) and there was clear congruence of gas exchange pattern with the simultaneous CNS measurement experiments (Fig. 3(a)). The routine establishment of DGE and similar total $\mathrm{CO}_{2}$ release during CNS recording are contrary to what would be associated with abnormal ventilation. Using ANOVA, mean cycles $\mathrm{h}^{-1}$ and $\mathrm{CO}_{2}$ burst duration were not significantly different between the respirometry-only and CNS experiments during DGE at $40 \%, 21 \%$ and $10 \%$ oxygen (d.f. $=1, F=0.03, p=0.86$; d.f. $=1, F=0.36$, $p=0.56$ ). Among all four different oxygen tensions, the mean total $\mathrm{CO}_{2}$ output rates were also not significantly different between the respirometry-only and CNS experiments (d.f. $=1, F=2.16, p=0.17$ ). Quantitative and qualitative comparisons thus show minimal impact of the dissection and electrode placement on gas exchange patterns.

At normoxia during CNS measurement, ventilation associated with the flutter phase during the inter-burst period and sawtoothing were observed as part of the typical DGE cycle (Figs. 2 and 3(a)). Sawtoothing has been previously described as fluttering during a $\mathrm{CO}_{2}$ burst by Duncan and Byrne (2000), and here it refers to small spikes of $\mathrm{CO}_{2}$ release associated with abdominal pumping movements during a burst. DGE was maintained in $P$. americana when cockroaches were exposed to hyperoxia or moderate hypoxia. In $40 \%$ oxygen, $\mathrm{CO}_{2}$ bursts were defined by an initial peak, followed by a drop in $V \mathrm{CO}_{2}$ due to the absence of sawtoothing and then a second peak at the end of the burst (Fig. 3(b)). In 10\% oxygen, DGE was maintained, but $V \mathrm{CO}_{2}$ was significantly elevated during the inter-burst period (Fig. 3(c), Table 1). The $\mathrm{CO}_{2}$ bursts in $10 \%$ oxygen usually had two release peaks in close succession at the start of the burst and then protracted $\mathrm{CO}_{2}$ release for the remainder of the burst. At $2 \%$ oxygen, all periodicity of $\mathrm{CO}_{2}$ release was lost and $V \mathrm{CO}_{2}$ remained relatively constant during exposure (Fig. 3(d)).

Rates of $\mathrm{CO}_{2}$ output at the four oxygen tensions during simultaneous CNS measurement are presented in Table 1. The total rate of $\mathrm{CO}_{2}$ output $\left(\mathrm{mL} \mathrm{h}^{-1}\right)$ was not significantly different at any of the oxygen tensions, suggesting the cockroaches maintain their oxygen requirements through ventilation. At $40 \%, 21 \%$ and $10 \%$ oxygen, where DGE was employed, $\mathrm{CO}_{2}$ output increased during the inter-burst period as oxygen tension decreased; however, $\mathrm{CO}_{2}$ output during a burst, burst period, and burst frequency were not significantly different between oxygen treatments (Table 1).

\subsection{Ventilatory CNS output}

CNS recordings were coupled with $\mathrm{CO}_{2}$ output and a range of gas exchange patterns were detected from continuous through to discontinuous cycles depending on 
Table 1

The effect of oxygen concentration on gas exchange in P. americana $\left(n=6\right.$ per treatment) at $25^{\circ} \mathrm{C}$ during simultaneous $\mathrm{CNS}$ measurement (mean $\left.\pm \mathrm{SD}\right)$

\begin{tabular}{|c|c|c|c|c|}
\hline Oxygen tension $(\%)$ & 40 & 21 & 10 & 2 \\
\hline Mass (g) & $0.984 \pm 0.090$ & $1.100 \pm 0.180$ & $1.121 \pm 0.151$ & $0.958 \pm 0.055$ \\
\hline No. of DGE cycles examined & 18 & 23 & 17 & - \\
\hline Total cycle $\mathrm{CO}_{2}$ output $\left(\mathrm{mLh}^{-1}\right)$ & $0.28 \pm 0.06$ & $0.30 \pm 0.05$ & $0.35 \pm 0.08$ & $0.29 \pm 0.05$ \\
\hline Burst $\mathrm{CO}_{2}$ output $\left(\mathrm{mL} \mathrm{h}^{-1}\right)$ & $0.45 \pm 0.12$ & $0.47 \pm 0.13$ & $0.44 \pm 0.09$ & - \\
\hline Burst period (min) & $8.7 \pm 2.4$ & $9.0 \pm 2.5$ & $9.2 \pm 2.5$ & - \\
\hline Burst frequency (bursts $\mathrm{h}^{-1}$ ) & 3.8 & 3.1 & $3.6^{-}$ & - \\
\hline Inter-burst $\mathrm{CO}_{2}$ output $\left(\mathrm{mL} \mathrm{h}^{-1}\right)$ & $0.10 \pm 0.06$ & $0.15 \pm 0.07^{*}$ & $0.21 \pm 0.09^{*}$ & - \\
\hline Inter-burst period (min) & $7.1 \pm 4.4$ & $10.4 \pm 4.9$ & $7.5 \pm 5.1$ & - \\
\hline DGE cycle period (min) & $15.8 \pm 5.9$ & $19.4 \pm 6.2$ & $16.7 \pm 9.4$ & - \\
\hline
\end{tabular}

In the absence of DGE cycles at $2 \%$ oxygen, the total cycle $\mathrm{CO}_{2}$ output value was calculated from a $1 \mathrm{~h}$ period when each individual was at rest.

* Indicates a significant difference between means in a given row (ANOVA, $p<0.05$ ).

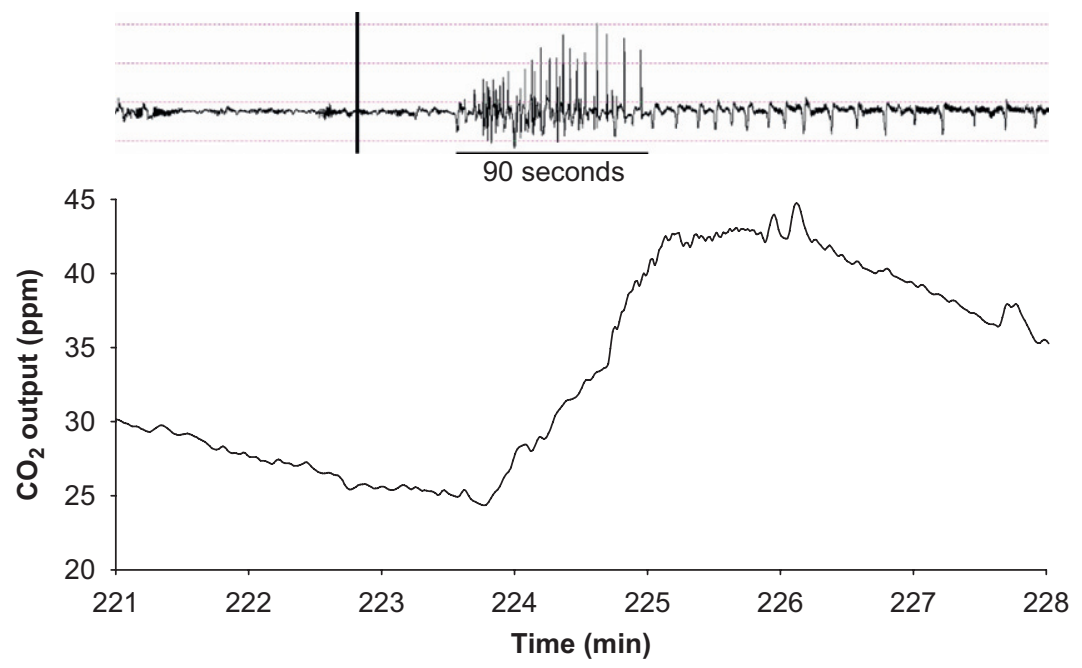

Fig. 4. A short section of representative $\mathrm{CNS}$ recording showing the initial nervous response (upper trace) and corresponding $\mathrm{CO}_{2}$ release to $2 \%$ oxygen after normoxia. The solid vertical line represents the switch to $2 \%$ oxygen.

oxygen tension (Fig. 3). Large CNS outputs during DGE cycles were consistently associated with maximal $\mathrm{CO}_{2}$ bursts (d.f. $=17, t=11.23, p<0.001$ ), where CNS output slightly preceded each $\mathrm{CO}_{2}$ release peak. At normoxia, large CNS outputs were detected at the beginning of the $\mathrm{CO}_{2}$ burst (and occasionally also later during the burst) and were followed by consistent, moderate CNS output associated with sawtoothing (Fig. 3(a)). During the interburst period, reduced CNS activity was observed immediately after the burst, followed by a return to moderate CNS activity during the flutter phase preceding the next $\mathrm{CO}_{2}$ burst.

The CNS output pattern was quite different under hyperoxic conditions; large CNS peaks were aligned with observed muscular abdominal pumping and these coincided consistently with the beginning and end of the $\mathrm{CO}_{2}$ burst (Fig. 3(b)). There were also no periods of moderate CNS output matching with $\mathrm{CO}_{2}$ sawtoothing or fluttering periods, as seen at normoxia. Other than $\mathrm{CO}_{2}$ burst-related CNS spikes, moderate CNS output was minimal at $40 \%$ oxygen. By contrast, at $10 \%$ oxygen, constant moderate CNS output was recorded in between the periodic large
CNS outputs. The large CNS outputs at $10 \%$ oxygen coincided with strong abdominal pumping movements and occurred consistently in close succession at the start of the $\mathrm{CO}_{2}$ burst (Fig. 3(c)). In 2\% oxygen, CNS output was largely constant at a moderate level and CNS bursts were not observed after the initial reaction to the gas (Fig. 3(d)). We observed only occasional small muscular ventilation movements during $2 \%$ oxygen exposure.

Within 60 s of switching from normoxia to $40 \%, 10 \%$ or $2 \%$ oxygen, each animal showed a CNS response to the change in oxygen tension, which coincided with a short series of abdominal movements. The initial CNS ventilatory response to $2 \%$ oxygen and the corresponding transient increase in $V \mathrm{CO}_{2}$ is shown in Fig. 4. The CNS response to a switch from normoxia to $40 \%$ or $10 \%$ oxygen was less intense and typically less than $1 \mathrm{~min}$ in duration, with no corresponding peak in $\mathrm{CO}_{2}$ release.

\section{Discussion}

Ventilation in insects is controlled by rhythmic nervous innervation that is influenced by metabolic, hormonal, 
sensory, neural, and behavioral factors (Bustami and Hustert, 2000; Nation, 2002). The technique used here efficiently records CNS ventilatory activity and is applicable to any medium to large sized terrestrial arthropod $(>0.5 \mathrm{~g})$ with ventral access to the nerve cord not requiring large dissection. Whilst in situ neurophysiological preparations have been available for some time (e.g. Case, 1961), this present method further minimizes the potential for invasive trauma and confers the ability to concurrently visualize standard resting patterns of $V \mathrm{CO}_{2}$. Indeed, for $P$. americana, previous surgical techniques for identifying ventilatory nervous output may have induced $\mathrm{CPG}$ shifting and interference with interganglionic control mechanisms (Farley et al., 1967).

For $P$. americana, $\mathrm{CO}_{2}$ output rates were maintained at all oxygen tensions examined, suggesting, under the assumption of a constant respiratory quotient, that oxygen uptake and metabolic rate are comparable across the range (Table 1). Although DGE was maintained under mild hypoxic conditions, inter-burst $\mathrm{CO}_{2}$ release was significantly elevated compared with normoxia. This is consistent with that reported for the adult ant, Camponotus vicinus, which increased flutter phase $V \mathrm{CO}_{2}$ by approximately $37 \%$ at $10 \%$ oxygen, compared to that at normoxia (Lighton and Garrigan, 1995). For P. americana we were unable to consistently distinguish the closed and flutter phases of DGE at each oxygen tension; however, for the combined inter-burst period we observed a similar $40 \%$ increase in $V \mathrm{CO}_{2}$ at $10 \%$ oxygen compared to that at normoxia. Interestingly, DGE cycle duration was not significantly different between normoxia and $10 \%$ oxygen in $P$. americana. For $C$. vicinus, hypoxia caused a marked increase in flutter phase duration, which increased DGE cycle duration and therefore decreased cycle frequency (Lighton and Garrigan, 1995). We propose that ventilatory activity during the flutter phase, in addition to oxygen uptake during the $\mathrm{CO}_{2}$ burst phase, is sufficient to meet oxygen requirements without lengthening of the flutter phase in $P$. americana. DGE was abandoned in $P$. americana at $2 \%$ oxygen for continuous gas exchange, as has been reported for another cockroach, Macropanesthia rhinoceros (Woodman et al., 2007).

Two extremes of CNS output pattern emerged during gas exchange measured in $P$. americana under different oxygen tensions. Under hyperoxic conditions, large CNS outputs were recorded at the beginning and end of each $\mathrm{CO}_{2}$ burst (Fig. 3(b)), which we interpret as co-ordinated opening of most of the spiracles and abdominal pumping to facilitate the bulk release of $\mathrm{CO}_{2}$. At other times there is minimal moderate nervous activity associated with each gas exchange cycle. By contrast, after the initial reaction (Fig. 4), exposure of P. americana to $2 \%$ oxygen results in largely continuous moderate CNS output (Fig. 3(d)) that coincided only occasionally with visible muscular ventilation movements. Large CNS output and $\mathrm{CO}_{2}$ bursts are absent during severe hypoxia and we assume that the spiracles remain largely open during exposure to maximize oxygen uptake.

At $10 \%$ oxygen, moderate CNS activity, punctuated by large CNS outputs at the beginning of each $\mathrm{CO}_{2}$ burst, is recorded throughout the gas exchange cycle (Fig. 3(c)) consistent with nervous activity to maintain oxygen uptake. Under normoxic conditions, large CNS output signaling the beginning of a $\mathrm{CO}_{2}$ burst is recorded (occasionally a second large CNS spike was observed later during the burst) with moderate CNS output during the sawtoothing and flutter phases of the DGE cycle (Fig. 3(a)).

From these comparisons, it appears that large CNS spikes represent co-ordinated spiracular opening and strong abdominal pumps to affect a $\mathrm{CO}_{2}$ burst during DGE, and moderate CNS activity is mainly associated with small abdominal movements (sometimes too small to be identified by the naked eye) and spiracular opening and closing during the flutter phase. Moderate CNS output thus most likely results in small-scale abdominal pumping as per the data presented for P. americana in Slama (1999) (termed 'concealed extracardiac pulsations'). Our results support $\mathrm{CO}_{2}$ bursts (in particular, sawtoothing) during DGE at normoxia, that would align with extracardiac pulsations facilitated by the nervous output shown in Fig. 3(a). Future measurement of hemocoelic pressure would be valuable in assigning CNS mediated abdominal movements to $\mathrm{CO}_{2}$ release for the oxygen tensions other than normoxia in Fig. 3.

As described above, hyperoxia resulted in minimal moderate CNS activity and a loss of $\mathrm{CO}_{2}$ sawtoothing and fluttering compared with that observed in normoxia and mild hypoxia. Sawtoothing and fluttering appear to represent important periods of oxygen uptake based on their absence at $40 \%$ oxygen and the nearly continuous flutter-type activity at $10 \%$ oxygen. We propose that the reduced moderate CNS activity in hyperoxia functions to avoid excessive oxygen uptake, in accordance with the oxygen toxicity hypothesis for DGE (Hetz and Bradley, 2005; Bradley, 2006). Exposure to $40 \%$ oxygen results in the elimination of the flutter phase, as oxygen uptake during this period would be unnecessary, given bulk uptake during the burst (Hetz and Bradley, 2005; Woodman et al., 2007). By contrast, we propose that gas exchange at $10 \%$ oxygen (Fig. 3(c)) represents an early stage of the degradation of DGE toward the continuous pattern at $2 \%$ oxygen, based on constant moderate CNS output during the cycle.

The partial pressure of oxygen in the tracheae is recognized as an important factor regulating the various gas exchange patterns among insects (Burkett and Schneiderman, 1967) and our results support this relative to CNS output from the metathoracic ganglion at different oxygen tensions for $P$. americana. As has been reported for other insects (e.g. Bustami et al., 2002), we infer the existence of localized oxygen receptors in the CNS for P. americana based on clear CNS response to changes in oxygen tension 
within $60 \mathrm{~s}$ of exposure (Fig. 4). Tracheae relay respiratory gases directly into the CNS ganglia (Nation, 2002; Bustami et al., 2002) and therefore, it is interesting that our results show a more immediate response to a change in atmospheric oxygen than that previously reported for a directly exposed isolated grasshopper CNS with the tracheae intact (Bustami et al., 2002). It was suggested that one existing oxygen levels within the tracheae considerably delay the response to a change in atmospheric oxygen. Whilst such an effect is not disputed, our live preparations suggest a lesser delay for $P$. americana, notwithstanding the different tracheal dimensions as a result of the preparation, intact spiracles, and body size. It is possible that a more immediate response to a change in oxygen tension is due to active ventilation in the intact live specimen more rapidly delivering air to the ganglion. Further research to identify the exact location and function of respiratory chemoreceptors would require more invasive and extensive specimen manipulation. For example, Myers and Retzlaff (1963) showed how removal of the cephalic nerves by decapitation immediately disrupted discontinuous ventilation in the Cuban burrowing cockroach. For this species, an oscillatory feedback mechanism between the abdominal ganglia is suppressed by the brain, allowing the CPG to release periodic bursts that result in discontinuous ventilation of the tracheae.

\section{Concluding remarks}

The technique presented here provides excellent potential for minimally invasive insight into the interactions between the CNS and whole-organism physiology. Specifically, we can determine how patterns of gas exchange are regulated in response to environmental variables such as atmospheres that deviate from normoxia. By real-time switching of experimental treatments and continuous simultaneous recordings, the activity of CPGs for ventilation can be assessed, in addition to inferring the presence of localized oxygen and/or carbon dioxide sensors at different points in the CNS. There is also applied potential in better understanding how different chemicals and drugs may affect the CNS and subsequently, ventilation and spiracular control (Woodman et al., in press).

Whilst insect respiratory physiology has re-emerged as a flourishing and spirited field of research (Quinlan and Gibbs, 2006), an underlying mechanistic understanding behind different modes of gas exchange has remained somewhat left behind. Here we permit for the first time the visualization of ventilatory CNS output relative to typical resting gas exchange regimes at different oxygen tensions.

\section{Acknowledgments}

For technical and engineering assistance we thank Greg Dojchinov and Stuart Alley (CSIRO Entomology). This research was financially supported by a $\mathrm{Ph} . \mathrm{D}$. scholarship awarded to J.D.W. by the Bulk Handling Companies of Australia.

All experiments as part of the research presented herein were conducted in compliance with the current laws of Australia.

\section{References}

Bradley, T.J., 2006. Discontinuous ventilation in insects: protecting tissues from $\mathrm{O}_{2}$. Respiratory Physiology and Neurobiology 154, 30-36.

Burkett, B.N., Schneiderman, H.A., 1967. Control of the spiracles in silk moths by oxygen and carbon dioxide. Science 156, 1604-1606.

Burrows, M., 1975. Co-ordinating interneurones of the locust which convey two patterns of motor commands: their connexions with ventilatory motoneurones. Journal of Experimental Biology 63, 735-753.

Burrows, M., 1982. Interneurones co-ordinating the ventilatory movements of the thoracic spiracles in the locust. Journal of Experimental Biology 97, 385-400.

Bustami, H.P., Hustert, R., 2000. Typical ventilatory pattern of the intact locust is produced by the isolated CNS. Journal of Insect Physiology 46, 1285-1293.

Bustami, H.P., Harrison, J.F., Hustert, R., 2002. Evidence for oxygen and carbon dioxide receptors in insect CNS influencing ventilation. Comparative Biochemistry and Physiology Part A 133, 595-604.

Case, J.F., 1961. Organisation of the cockroach respiratory centre. Biological Bulletin 121, 385.

Chown, S.L., Gibbs, A.G., Hetz, S.K., Klok, C.J., Lighton, J.R.B., Marais, E., 2006. Discontinuous gas exchange in insects: a clarification of hypotheses and approaches. Physiological and Biochemical Zoology 79, 333-343.

Duncan, F.D., Byrne, M.J., 2000. Discontinuous gas exchange in dung beetles: patterns and ecological implications. Oecologia 122, 452-458.

Farley, R.D., Case, J.F., Roeder, K.D., 1967. Pacemaker for tracheal ventilation in the cockroach, Periplaneta americana (L.). Journal of Insect Physiology 13, 1713-1728.

Gray, E.M., Bradley, T.J., 2006. Evidence from mosquitoes suggests that cyclic gas exchange and discontinuous gas exchange are two manifestations of a single respiratory pattern. Journal of Experimental Biology 209, 1603-1611.

Hetz, S.K., Bradley, T.J., 2005. Insects breathe discontinuously to avoid oxygen toxicity. Nature 433, 516-519.

Kestler, P., 1985. Respiration and respiratory water loss. In: Environmental Physiology and Biochemistry of Insects. Springer, Berlin, pp. 137-184.

Lewis, G.W., Miller, P.L., Mills, P.S., 1973. Neuro-muscular mechanisms of abdominal pumping in the locust. Journal of Experimental Biology 59, 149-168.

Lighton, J.R.B., 1996. Discontinuous gas exchange in insects. Annual Review of Entomology 41, 309-324.

Lighton, J.R.B., Garrigan, D., 1995. Ant breathing: testing regulation and mechanism hypotheses with hypoxia. Journal of Experimental Biology 198, 1613-1620.

Marais, E., Chown, S.L., 2003. Repeatability of standard metabolic rate and gas exchange characteristics in a highly variable cockroach, Perisphaeria sp. Journal of Experimental Biology 206, 4565-4574.

Marder, E., Bucher, D., 2001. Central pattern generators and the control of rhythmic movements. Current Biology 11, R986-R996.

Mill, P.J., Hughes, G.M., 1966. The nervous control of ventilation in dragonfly larvae. Journal of Experimental Biology 44, 297-316.

Miller, P.L., 1960. Respiration in the desert locust. I. The control of ventilation. Journal of Experimental Biology 37, 224-236.

Miller, P.L., 1966. The regulation of breathing in insects. Advances in Insect Physiology 3, 279-354. 
Myers, T., Retzlaff, E., 1963. Localisation and action of the respiratory centre of the Cuban burrowing cockroach. Journal of Insect Physiology 9, 607-614.

Nation, J.L., 2002. Insect Physiology and Biochemistry. CRC Press, Boca Raton, FL.

Quinlan, M.C., Gibbs, A.G., 2006. Discontinuous gas exchange in insects. Respiratory Physiology and Neurobiology 154, 18-29.

Ramirez, J.M., Pearson, K.G., 1989. Distribution of intersegmental interneurones that can reset the respiratory rhythm of the locust. Journal of Experimental Biology 141, 151-176.

Reinhold, K., 1999. Energetically costly behaviour and the evolution of resting metabolic rate in insects. Functional Ecology 13, 217-224.

Slama, K., 1999. Active regulation of insect respiration. Annals of the Entomological Society of America 92, 916-929.
Wasserthal, L.T., 1996. Interaction of circulation and tracheal ventilation in holometabolous insects. Advances in Insect Physiology 26, 297-351.

Woodman, J.D., Cooper, P.D., Haritos, V.S., 2007. Cyclic gas exchange in the giant burrowing cockroach, Macropanesthia rhinoceros: effects of oxygen tension and temperature. Journal of Insect Physiology 53, 497-504.

Woodman, J.D., Haritos, V.S., Cooper, P.D., in press. Effects of phosphine on the neural regulation of gas exchange in Periplaneta americana. Comparative Biochemistry and Physiology Part C.

Zafeiridou, G., Theophilidis, G., 2006. A simple method for monitoring the respiratory rhythm in intact insects and assessing the neurotoxicity of insecticides. Pesticide Biochemistry and Physiology $86,211-217$. 